Vier Beispiele aus der Praxis

\title{
Nachhaltige Hochschulbildung in der Umsetzung
}

Die Hochschulen stehen vor großen Herausforderungen. Doch wie gehen sie in der Praxis mit den veränderten Rahmenbedingungen um? Vier Praxisbeispiele zeigen die Bandbreite verschiedener Ausbildungsstrukturen, die der Bologna-Prozess für die Verankerung des Leitbilds der nachhaltigen Entwicklung in der Hochschullehre bietet.

\section{Ausbildung zum Manager der Nachhaltigkeit}

\begin{abstract}
An der Universität Oldenburg startet ein neuartiger Master-Studiengang, der auf die Vermittlung von wirtschaftswissenschaftlichen und fachübergreifenden Qualifikationen zur Umsetzung von Nachhaltigkeit abzielt.
\end{abstract}

M it dem Ziel „Manager/-innen der Nachhaltigkeit“ auszubilden, startet die Universität Oldenburg ab dem Wintersemester 2006/07 den Masterstudiengang Sustainability Economics and Management (SEM). Er soll Absolventen/-innen die fachlichen, analytischen, inderdisziplinären und sozialen Kompetenzen an die Hand geben, um ihnen eine Tätigkeit in Unternehmen, Behörden, Nichtregierungsorganisationen (NGOs) oder internationalen Organisationen zu ermöglichen.

Die Universität Oldenburg baut dabei auf den Erfahrungen des seit 1997 bestehenden Diplomstudiengangs Wirtschaftswissenschaften mit ökologischem Schwerpunkt auf. Mit der Einführung von Bachelor und Masterstudiengängen wurde die Chance genutzt, ein spezifisches Masterangebot zu erstellen, was eine noch stärkere Spezialisierung im Hinblick auf Berufsfelder ermöglicht.

Der Studiengang ist eng verknüpft mit der Umwelt- und Nachhaltigkeitsforschung an der Universität und ist eingebunden in eine neu entstehende Zentrumsstruktur. Neben den Wirtschaftswissenschaften und dem Bereich Recht ist auch die Informatik beteiligt. Zudem gib es ein breites Spektrum von Anknüpfungspunkten in der Physik, der Biologie, den Meeres- wissenschaften und der Umwelt- und Raumplanung. Dadurch kann der nötigen Interdisziplinarität Rechnung getragen werden, welche die mehrdimensionalen Probleme der Nachhaltigkeit erfordert (siehe Beitrag von Schneidewind/Dettleff in diesem Heft). An der Universität entstehen zurzeit Masterangebote mit stark naturwissenschaftlichem Fokus, welche zu einem umweltbezogenen Master-Cluster zusammengefasst werden, um so die Wahlmöglichkeiten weiter auszubauen. Dies soll mit innovativen Lehrkonzeptionen wie Projekten, Planspielen und praktischen Studien geschehen. Dabei wird auch auf eine enge Einbindung von nationalen und internationalen Organisationen sowie ausländischen Partneruniversitäten Wert gelegt.

Nach einer Regelstudienzeit von vier Semestern wird der Abschluss Master of Arts (M.A.) erworben. Studienbeginn ist jeweils zum Wintersemester. Im ersten Semester sind verpflichtend Module in den Bereichen Umwelt- und Ressourcenökonomie, Nachhaltigkeitspolitik und -management, Umweltrecht sowie Umwelt- und Geowissenschaften zu belegen. Im zweiten Semester erfolgt eine Vertiefung dieser Module, ergänzend kommt ein Modul zur Corporate Social Responsibility hinzu. Des Weiteren wird eine der folgenden Spezialisierungsrichtungen gewählt:

- Erneuerbare Energien

- Umwelt- und Raumplanung

I Umweltinformatik

- Marketing

- Innovationsmanagement und Entrepreneurship

Im dritten Semester muss zusätzlich eine Veranstaltung zur Klimaschutzökonomik belegt und in Gruppenarbeit ein Forschungs- oder Praxisprojekt zum Nachhaltigkeitsmanagement durchgeführt werden. Im zweiten und dritten Semester können Wahlpflichtmodule teils für Sprachen, teils für ein beliebiges Fach, auch aus anderen Fachdisziplinen belegt werden. Ebenfalls besteht im zweiten oder dritten Semester die Möglichkeit eines Auslandssemesters bei einer der Partneruniversitäten mit ähnlichen Studiengängen. Das vierte Semester beinhaltet ein Kolloquium zur Forschungsmethodik. 
Für Absolventen(innen) des Masters Sustainability Economics and Management ergeben sich vielfältige Berufschancen, insbesondere in Kommunikations-, Umwelt- und Nachhaltigkeitsabteilungen oder bei speziellen Projektvorhaben wie beispielsweise der Einführung von Umweltmanagementsystemen in großen Unternehmen. Aber auch kleinere und mittlere Unternehmen, zum Beispiel jene aus der stetig wachsenden Branche der regenerativen Energien suchen spezifisch ausgebildete Kräfte. Berater- oder Gutachtertätigkeiten sind ein weiteres Berufsfeld. Dazu kommt ein steigender Bedarf in verschiedenen internationalen Organisationen, NGOs und Behörden auf nationaler und auf EU-Ebene. Ähnlich ist die Situation in der Wissenschaft in der immer mehr nachhaltigkeitsorientierte Forschungsvorhaben gefördert werden.

In Abhängigkeit von den jeweils gewählten Vertiefungsrichtungen können sich Absolventen(innen) des Studiengangs in Richtung der folgenden Berufsprofile und Beschäftigungsfelder qualifizieren:

- Manager(in) integrativer Lösungsprozesse und nachhaltigkeitsorientierter Projekte in Unternehmen, Nicht-Regierungsorganisationen, Stiftungen oder in der Politikberatung; zum Beispiel in Stabsabteilungen großer Unternehmen, als Manager(in) unternehmensinterner Projekte und unternehmensübergreifender Initiativen zur Nachhaltigkeit oder als Campaigner(in) bei Umweltverbänden

- Manager(in) von nachhaltigkeitsorientierten Finanzanlagen und Versicherungen. Beispiele wären der Sustainability Dow Jones oder Rückversicherungen gegen Naturkatastrophen

- Internationale(r) Manager(in) und Sachverständige(r), beispielsweise im Bereich internationaler Organisationen oder in international tätigen Unternehmen

- Berater(in) für Unternehmen im Bereich der Umsetzung von nachhaltigkeitsorientierten Assessments und des $\mathrm{CO}_{2^{-}}$ Zertifikatehandels oder für die kommunale, regionale oder nationale Politik

【 Ökonomische(r) Modellierer(in) mit umweltökonomischer oder integrierter Ausrichtung (Integrated Assessment Modelling)

- Wissenschaftler(in) mit disziplinübergreifender Nachhaltigkeitskompetenz

- Mitarbeiter(in) in EU-Einrichtungen oder in EU-nahen Organisationen, beispielsweise in der Anwendung von EURecht, der Beratung von EU-Institutionen und von Mitgliedsstaaten mit einem Fokus auf Umwelt- und Nachhaltigkeitsthemen. Durch die EU-Erweiterung ist hier ebenfalls ein wachsender Bedarf zu erwarten.

Es wird angestrebt neben einer starken internationalen Ausrichtung auch eine enge Einbindung potenzieller Arbeitgeber, zum Beispiel durch Planspiele, Vorträge, Projekte oder Prakti$\mathrm{ka}$ in den Masterstudiengang herzustellen.

\section{Anmerkungen:}

Weitere Informationen unter www.sustainability.uni-oldenburg.de
I AUTOREN + KONTAKT

Dr. Bernd Siebenhüner ist Juniorprofessor für Ökologische Ökonomie an der Carl von Ossietzky Universität Oldenburg und Leiter der Nachwuchsforschungsgruppe Gesellschafliches Lernen und Nachhaltigkeit (GELENA) sowie Koordinator des Studiengangs MA "Sustainability Economics and Management".

PD Dr. Martin Müller verwaltet den Lehrstuhl für Produktion und Umwelt an der Carl von Ossietzky Universität Oldenburg und ist Koordinator des Studiengangs MA "Sustainability Economics and Management".

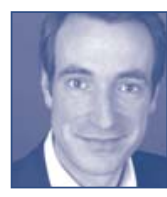

Carl von Ossietzky Universität Oldenburg, Fakultät 2: Informatik, Wirtschafts- und Rechtswissenschaften, 26111 Oldenburg. E-Mail: martin.mueller@uni-oldenburg.de; bernd.siebenhuener@uni-oldenburg.de

\section{Nachhaltigkeit im Fokus interdisziplinärer Studienschwerpunkte}

\section{An der Technischen Universität Darmstadt werden drei der interdisziplinären Studien- schwerpunkte inhaltlich auf die Nachhaltigkeits- thematik bezogen. Im Hinblick auf die Vermitt- lung von Schlüsselkompetenzen geschieht dies methodisch-didaktisch. Organisationell findet eine Verknüpfung durch Aktivitäten der beteilig- ten Partner statt.}

D s hier vorgestellte Projekt Sustainable Technology Education Program (STEP) ist eines der wenigen Projekte im Rahmen der UN-Dekade 2005-2014 Bildung für nachhaltige Entwicklung,welches im Bereich der Hochschulbildung als offizielles Dekadeprojekt ausgezeichnet wurde. Nachhaltigkeit steht bei STEP auf zwei Weisen im Fokus der Hochschullehre, sowohl als Gegenstand als auch als Zielperspektive.

Basis des Projektes sind drei der interdisziplinären Studienschwerpunkte, welche an der Technischen Universität (TU) Darmstadt eine lange Tradition haben. Dies sind Umweltwissenschaften (UWS), Technologie und internationale Entwicklung (TuE) und Nachhaltige Gestaltung von Technik und Wissenschaft $(\mathrm{NaG})$. Alle drei sind thematisch fokussierte Lehr- \& Lernangebote, die sich an Studierende der Ingenieurwissenschaften der TU Darmstadt, aber auch an Studierende aller anderen Fachbereiche richten. Sie werden vom Zentrum für Interdisziplinäre Technikforschung (ZIT) und der Interdisziplinären Arbeitsgruppe Naturwissenschaft, Technik und Sicherheit $\rightarrow$ 
(c) 20I0 Authors; licensee IÖW and oekom verlag. This is an article distributed under the terms of the Creative Commons Attribution Non-Commercial No Derivates License (http://creativecommons.org/licenses/by-nc-nd/3.o/), which permits unrestricted use, distribution, and reproduction in any medium, provided the original work is properly cited. 